\title{
Low Cost Device for Charging Mobile Phone using Another Smartphone
}

\author{
Vaibhav Aggarwal ${ }^{1}$, Vaibhav Gupta $^{1}$, Kiran Sharma ${ }^{2 *}$, and Neetu Sharma ${ }^{3}$ \\ ${ }^{1}$ Department of Computer Science Engineering, Graphic Era University, Dehradun, Uttarakhand, India \\ ${ }^{2}$ Department of Physics, Graphic Era University, Dehradun, Uttarakhand, India \\ ${ }^{3}$ Department of Chemistry, Graphic Era University, Dehradun, Uttarakhand, India
}

Received January 22, 2018; Accepted March 15, 2018; Published May 1, 2018

\begin{abstract}
Mobile has been and will always remain one of the best companions for any human being. Mobile phones are considered as the live example of the advancement in technology on a daily basis. This era is marked by our complete dependence on this technology. The growing technology has introduced mobile phone, which plays an important role in communication. Since mobile phones have been made to be with the user all day and to carry out all the basis and high-performance task as per the demand of the user, the batteries need to be charged multiple times during a day. This imposes a burden on the user to carry a power bank while travelling; at times it becomes difficult if the power bank battery also drains out. This paper presents a small technique which may reduce this problem. The major components of the design are a capacitor of $2200 \mu \mathrm{F}$ at $5.63 \mathrm{~V}$ and LED $1.5 \mathrm{~V}$. The experimental data shows that the charging level of a mobile battery of $2100 \mathrm{mAh}$ can be enhanced from $10-19 \%$ in 35 minutes by consuming only $10 \%$ of the total energy of the other smart phone of battery $4000 \mathrm{mAh}$. Another experimental data shows that the charging level of a mobile battery of $2000 \mathrm{mAh}$ can be enhanced from $14-37 \%$ in 60 minutes by consuming only $20 \%$ of the total energy of the other smart phone of battery 4000 mAh. This low cost and simple designed USB On-the-Go (OTG) extension can now replace the necessity of carrying a power bank while travelling, which is expensive as compared to the above proposed technique as well.
\end{abstract}

Keywords: Mobile to mobile; Charging; On the Go (OTG); Low cost; Cable; Overcharging; Safe

\section{Introduction}

The advancement in science and technology has made mobile phones as an essential part in our life. Mobile phones have connected people living across the globe and had become a necessity in this advanced world. Due to the rapid increase functionality of mobile phone, the battery needs to be charged multiple times a day and moreover it cannot meet the increasing power demand [1,2]. It becomes difficult to charge the battery of our mobile phone while traveling from place to place. Ali et al. [3] have proposed a portable mobile phone charger for travelling but it consists of a multidirectional wind turbine which can only be used in motorcycles. Manikandan et al. [4] created an energy storage system for mobile phone using super capacitors which can be charged in few seconds. This can be used to replace the batteries in the mobile phone, 
but most cell phones and electronic gadgets are powered by batteries [4]. Power banks have also been used as an extendable source of energy for mobile phones [5]. Many workers [6-9] have used renewable energy sources as the source to charge the mobile phone but this technique of charging mobile phones is not successful while travelling. Rewaskar and Datar [10] investigated a method of charging mobile phone with microwaves, but it can only be implemented within the microwave range transmitted from transmitter using antennas at a frequency is $2.45 \mathrm{GHz}$. Also wireless transmission causes harmful effect on human body and this method is very expensive. Varadarajan [11] used a coin-based technique as battery chargers. However, this technique requires predefined values and a suitably designed micro-controller for mobile phone of various configurations. Grid power or a solar energy [11] is used as source for charging mobile phone, which will not be convenient for users during travelling. A combination of wind power, human power and electrical power [12] have been used to charge mobile phone while traveling, but it can only be used with wind turbines for instant charging using a geared DC generator. Cao and Yin [13] introduced a USB protocol On the Go (OTG) chip for protocol transform high speed data transfer with the contradiction of duplicative function of data compression board in the USB transfer. The charging level can be increased by increasing the current in our circuit. For that we are going to use current booster circuit, and we will be able to apply fast charging technique. As we know that the power bank is little bit expensive and needs some space to carry. But the circuit we have made is cheaper as compared to the power bank and can be easily carried around. The power bank needs to be charged first and then to be used, but the circuit we had made can be used instantly. Moreover, we need not to carry around another mobile for this purpose, instead we can ask our neighbors to share their mobile charge which is more economical. While charging one phone from another phone, the current, voltage and charging percentages that show the enhancement are constantly checked.

In a portable device, the enhancement is greater, as the current in power bank is at higher rating. So, for the enhancement of that, we would apply fast charging technique. Various techniques have been proposed [1-13] to charge mobile phones while travelling Mobiles have to be put to recharge after the batteries drain out. Normally the user carries a power bank; but at times it becomes difficult if the power bank battery also drains out.

\section{Materials and Methods}

\section{Circuit Design}

The design of this circuit keeps it easy to carry and use it (Figure 1). We have used the On the Go (OTG) extension to take the energy or the power from the other smart phone that we carry along and installed a circuit at the other end in order to make it more efficient and reliable. A-device acts as a USB host with the B-device acting as a USB peripheral. The host and peripheral modes may be exchanged later by using Host Negotiation Protocol (HNP). If an uncharged capacitor $\mathrm{C}$ is connected across the terminals of a battery of voltage $\mathrm{V}$, the transient current flows as the capacitor plates charge up. However, the current stops flowing as soon as the charge Q on the positive plate reaches the value $\mathrm{Q}=\mathrm{CV}$. At this point, the electric field between the plates cancels the effect of the electric field generated by the battery, and there is no further movement of charge. Thus, if a capacitor is placed in a DC circuit then, as soon as its plates have charged up, the capacitor effectively behaves like a break in the circuit. 


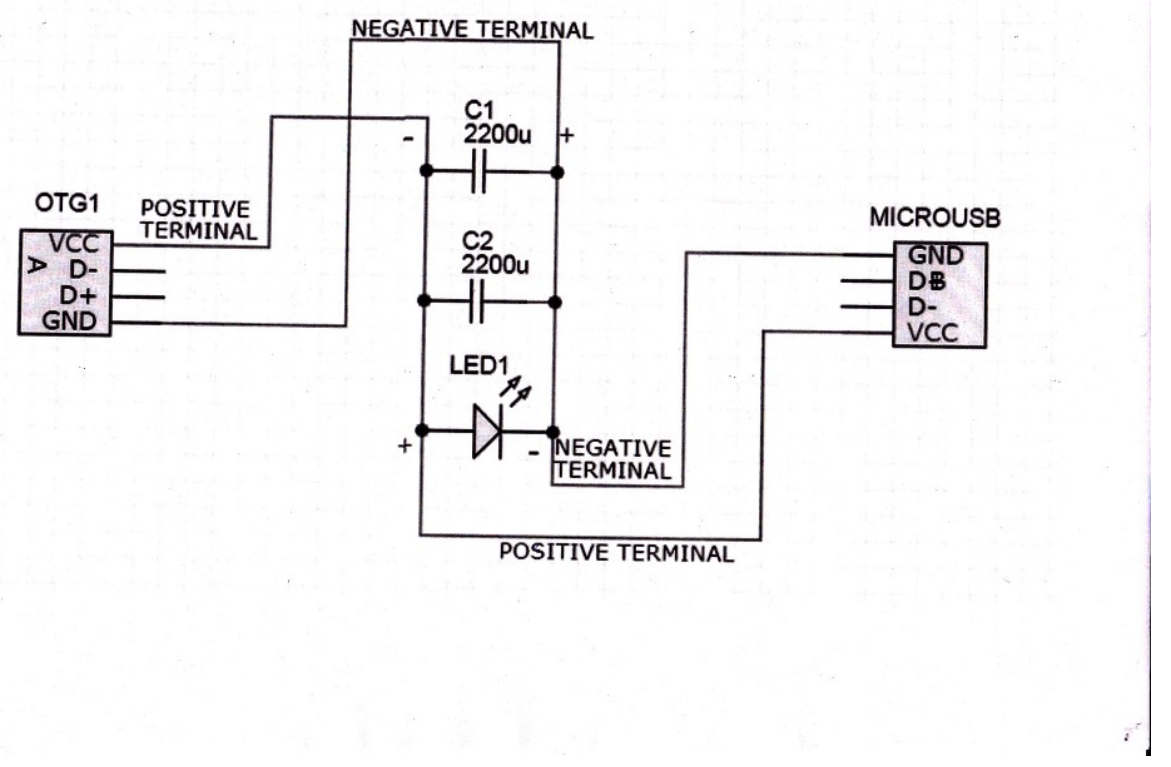

Fig. 1. Circuit design of OTG cable

The OTG cable can be used as the source to charge the mobile phones and also transfer high speed data [13]. This cheap, compact, and miniature circuit can be carried in pocket or bag and is light weight to carry it around. We took the required components and placed them on the printed circuit board (PCB) board as shown in the circuit diagram. Two capacitors of 2200 micro farad are connected in parallel. A since pin is used which is connected to the ground that helps in reverse charge or extract charge from the battery which is transferred to the other cell phone. The OTG cable is used to connect the mobile phone to the circuit having two capacitors connected in parallel. The charge flows through the OTG extension to the capacitors of $220 \mu$ Farad, which are connected in parallel. The capacitors are connected in parallel in order to increase its capacitance to increase the safety of this device. Now once the charge flows through the capacitors it meets an LED of $1.5 \mathrm{~V}$ in the circuit, which is connected in parallel to the capacitors and helps determine the status of the circuit at the input end. It will depict whether our circuit is completely error free or it needs some rectifications. Now once the charge flows through the LED it will pass on through the micro USB cable attached at the other end in order to power other devices.

\section{EXPERIMENTAL RESULTS \& DISCUSSION}

The designed OTG cable is used to charge the mobile phone from another smart phone. A mobile of $4000 \mathrm{mAh}$ has been used to power a Samsung phone of $2100 \mathrm{mAh}$ having $10 \%$ battery backup. It has been observed that in 30 min the battery of the phone can be enhanced up to $19 \%$ with only a loss of $20 \%$ charge of phone used at $5.63 \mathrm{~V}$ and $0.48 \mathrm{~A}$. The charging and discharging percentage with time of both phones is represented in Figure 2. 
Another set of phones are used. The charging time has been increased to $60 \mathrm{~min}$, and the mobile phone charged is $2000 \mathrm{mAh}$ and has 14\% battery backup. The charging and discharging \% with time of both the phone used has been shown in Figure 3 . The results show that in 60 min the battery of the phone can be enhanced up to $34 \%$ with only a loss of $20 \%$ charge of phone of $4000 \mathrm{mAh}$ used at $4.97 \mathrm{~V}$ and $0.44 \mathrm{~A}$.

The proposed OTG cable has also been used to charge a completely discharged mobile phone of $1050 \mathrm{mAh}$ for $60 \mathrm{~min}$. The experimental results show that a very less battery discharge has been observed from $63 \%$ to $57 \%$ in a mobile phone of $4100 \mathrm{mAh}$ used at $4.87 \mathrm{~V}$ and $0.54 \mathrm{~A}$. It has been observed that in $60 \mathrm{~min}$ the battery of the phone (Nokia-103) can be enhanced up to $31 \%$. Figure 3 shows the graphical representation of charging and discharging $\%$ of both phones used with time. The loss of charge can be calculated by constantly checking of the current, voltage and charging percentages of another phone by using multimeter. The loss of charge is same as we have used the same mobile phone for charging other mobile phones so the loss in percentage is same as compared other.

From Figures 2-3, it is clear that the battery of a mobile can be increased up to $37 \%$ in 60 min with negligible loss in charging of the other smart phone used.

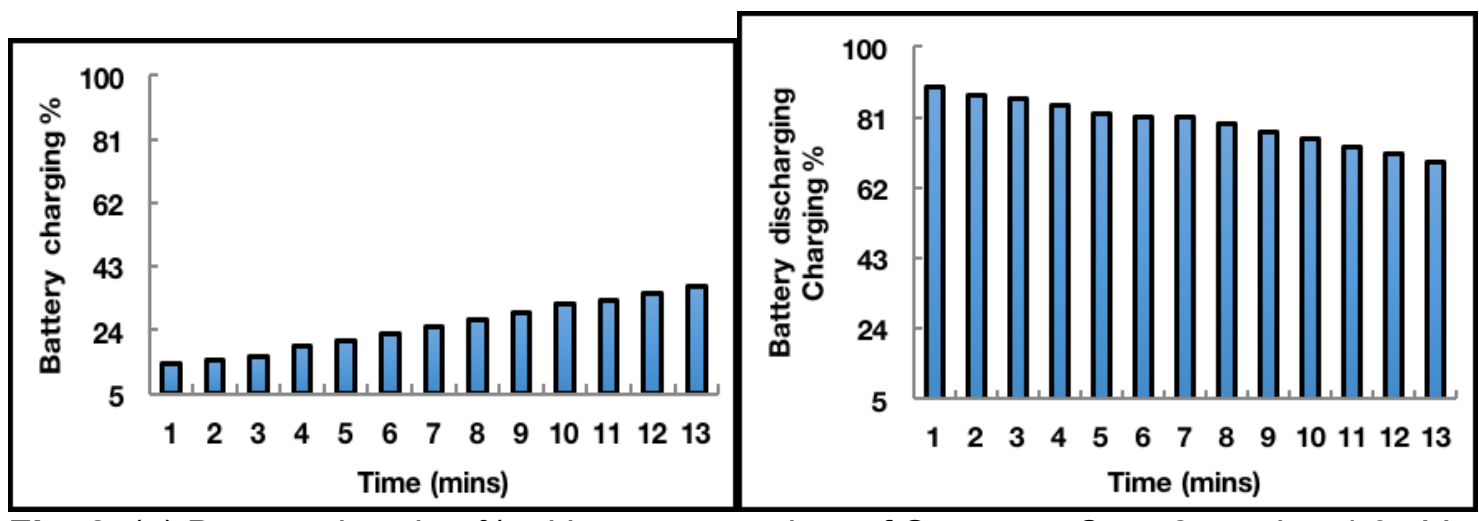

Fig. 2. (a) Battery charging \% with respect to time of Samsung Core-2 used at $4.97 \mathrm{~V}$ and $0.44 \mathrm{~A}$

(b) The battery discharging \% with respect to time of the smart phone used to drain charge.

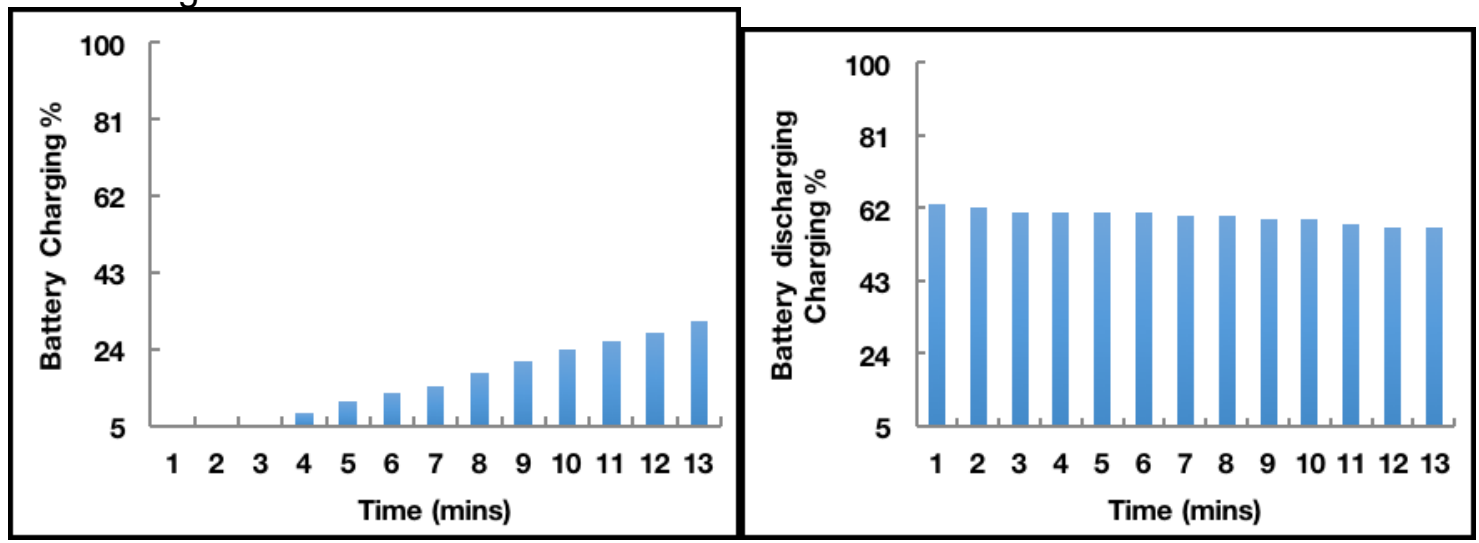

Fig. 3. (a) Battery charging $\%$ with respect to time of Nokia-103 used at $4.87 \mathrm{~V}$ and $0.54 \mathrm{~A}$

(b) The battery discharging \% with respect to time of the smart phone used to drain charge. 


\section{CONCLUSIONS}

In this paper, a low-cost method of charging mobile batteries has been designed for travelers, or people of rural and remote areas where the current supply is not available all the time. Now the necessity of carrying a power bank while travelling is also relaxed. This OTG is very useful in today's life because now days the necessity of communication is very important especially while moving from one place to another. Travelers can easily charge their phones by using this OTG cable simply by borrowing a Smartphone from the other passengers for few minutes. The proposed OTG cable is compact, simply designed and cheap.

\section{CONFLICTS OF INTEREST}

The authors declare that there is no conflict of interests regarding the publication of this paper.

\section{REFERENCES}

[1] S.S. Shivakumar, K.N.S. Mohana, "Ziziphus mauritiana leaves extracts as corrosion inhibitor for mild steel in $\mathrm{H}_{2} \mathrm{SO}_{4}$ and $\mathrm{HCl}$ solutions", European Journal of Chemistry, 3(4), pp. 426-432, 2012.

[2] P. Saikumar, D. Thamaraikanna, G. Yuvaraj, C. Yuvaraj, "Wind energy based mobile phone battery charging and battery application", International Journal for Research and Development in Engineering, pp. 6-11, 2014.

[3] K. Ali, W. S. W. Mohd, D. Rifai, M.I. Ahmed, A. Muzzakir, and T.A. Asyraf, "Design and Implementation of Portable Mobile Phone Charger Using Multidirectional Wind Turbine Extract", Indian Journal of Science and Technology, 9(9), pp. 1-6, 2016. DOI: 10.17485/ijst/2016/v9i9/88711

[4] J. Manikandan, R.M. Priya, S. Pavithra, D. Sarathkumar, "Rapid Smart Phone Charging Using Super Capacitor", International Journal of Advanced Research in Electrical, Electronics and instrumentation Engineering, 4(4), pp. 2175-2180, 2015.

[5] A. Joshi, "Low Cost, Portable and Extendable Power Bank", International Journal of Scientific \& Engineering Research, 6(4), pp. 87-89, 2015.

[6] S.B. Dhal, A. Agarwal, K. Agarwal, "Solar Powered Mobile Power Bank System", American Journal of Electrical and Electronic Engineering, 4(5), pp. 148-151, 2016.

[7] R. Kamble, S. Yerolkar, D. Shirsath, B. Kulkarni, "Solar Mobile Charger", International Journal of Innovative Research in Computer Science \& Technology, 2(4), 35-39, 2014.

[8] T. Singh, P. Bhardwaj, B. Singh, A. Kumar, "Design and development of Portable Power Charger: A Green Energy Initiative", International Journal of Electronics and Communication Technology, 5(4), pp. 12-15, 2014.

[9] A.N. Maroma, "Solar Powered Cell Phone Charging Station", Open Access Library Journal, 1(e1156), pp. 1-7, 2014. 
[10] P.A. Rewaskar, D. Datar, "Wireless Charging of Mobile Phone Using Microwave", International Journal of Computer Science and Mobile Computing, 3(4), pp. 427432, 2014.

[11] M.S. Varadarajan, "Coin Based Universal Mobile Battery Charger”, IOSR Journal of Engineering, 2(6), pp. 1433-1438, 2012.

[12] N.R.R. Reddy, Y. Sreekanth, M. Narayana "Mechanical and Electrical mobile charger", Int. Journal of Engineering Research and Applications, 3(6), pp. 1705 1708, 2013.

[13] M. Cao, P. Yin, "Research of USB OTG Technology in Image High-Speed Data Transfer”, Advanced Materials Research, 951, pp. 261-264, 2014.

Article copyright: (C) 2018 Vaibhav Aggarwal, Vaibhav Gupta, Kiran Sharma, and Neetu Sharma. This is an open access article distributed under the terms of the Creative Commons Attribution 4.0 International License, which permits unrestricted use and distribution provided the original author and source are credited.

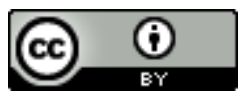

